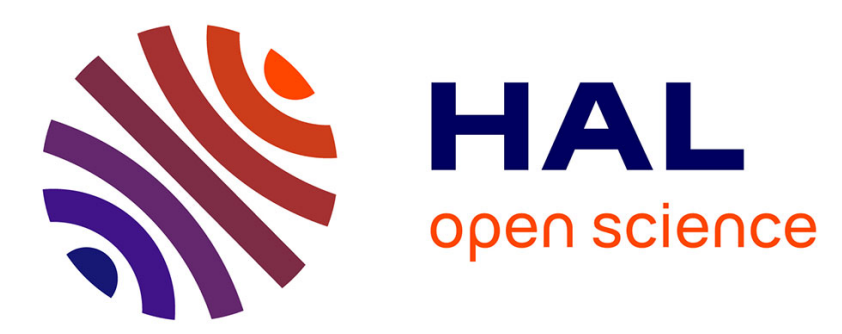

\title{
Cavity Modes Inside a Mode-Stirred Reverberation Chamber Extracted Using the Matrix Pencil Method
}

Francois Sarrazin, Elodie Richalot

\section{To cite this version:}

Francois Sarrazin, Elodie Richalot. Cavity Modes Inside a Mode-Stirred Reverberation Chamber Extracted Using the Matrix Pencil Method. 11th European Conference on Antennas and Propagation (EuCAP), Mar 2017, Paris, France. hal-01527192

\section{HAL Id: hal-01527192 \\ https://hal.science/hal-01527192}

Submitted on 24 May 2017

HAL is a multi-disciplinary open access archive for the deposit and dissemination of scientific research documents, whether they are published or not. The documents may come from teaching and research institutions in France or abroad, or from public or private research centers.
L'archive ouverte pluridisciplinaire HAL, est destinée au dépôt et à la diffusion de documents scientifiques de niveau recherche, publiés ou non, émanant des établissements d'enseignement et de recherche français ou étrangers, des laboratoires publics ou privés. 


\title{
Cavity Modes Inside a Mode-Stirred Reverberation Chamber Extracted Using the Matrix Pencil Method
}

\author{
Francois Sarrazin and Elodie Richalot \\ Université Paris-Est, ESYCOM (EA 2552), UPEMLV, ESIEE-Paris, CNAM, F-77454 Marne-la-Vallée, France \\ francois.sarrazin@u-pem.fr
}

\begin{abstract}
This paper presents the extraction of the modes resonating inside a mode-stirred reverberation chamber using the Matrix Pencil method. An increasing time-window technique is investigated as a process to discriminate the true modes and the spurious ones that appear due to the measurement noise.
\end{abstract}

Index Terms-Matrix Pencil, resonance frequency, reverberation chamber.

\section{INTRODUCTION}

Mode-Stirred Reverberation Chambers (MSRCs) are widely used in a variety of measurements like Electromagnetic Compatibility tests and antenna characterization [1]. The accuracy of such measurements depends directly on the MSRC properties. Indeed, the stirrers must provide a statistically (over a revolution) homogeneous and isotropic field distribution within the working volume. The minimum frequency from which this well-operating is attained, namely the Lower Useable Frequency, depends on the cavity geometry and needs to be determined experimentally. The classical measurement process to assess this frequency limit consists in evaluating uniformity and anisotropy criteria defined by the International Standard [2]. As they require field measurements at eight different locations of the receiving antenna, this implies human handling and lengthens the measurement process. Moreover, we showed the sensitivity of the estimated standard criterion to the exact antenna location and thus to the experimenter [3]. Therefore, we proposed a new criterion to characterize the MSRC behavior that is based on the cavity spectral properties and only requires measurements at a single position of the receiving antenna [4]. Indeed, from the measured transmission coefficient between two antennas located within the MSRC, we evaluate the distribution of the frequency spacings between resonance frequencies. The precise extraction of the later ones is thus necessary; moreover, as we calculate the spacings between adjacent frequencies, the non-detection of a few resonances would have an impact on the aimed distribution.

Different post-processing methods have been used to extract the resonance properties from the measured transmission coefficient both in time (Harmonic Inversion method [5]) and frequency domain (Vector Fitting Algorithm [6]). However, due to the high density of resonances and the overlap between them around and above the Lower Useable Frequency, we noticed the difficulty of extracting all the physical resonances and eliminating the spurious ones

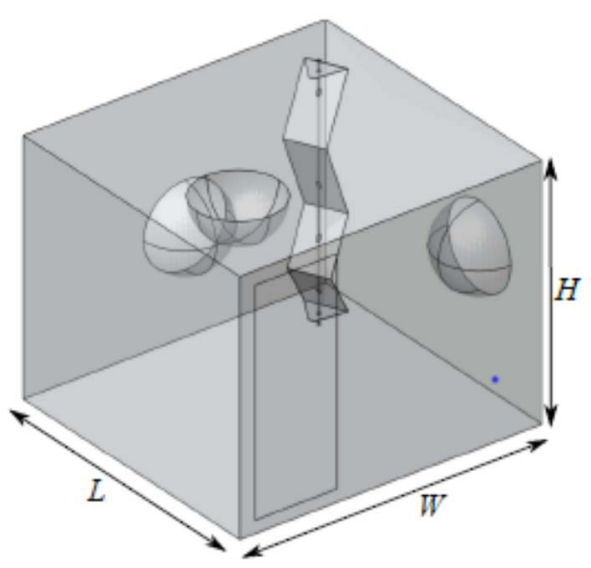

Fig. 1. Picture of the Mode-Stirred Reverberation Chamber $(\mathrm{W}=2.95 \mathrm{~m}$, $\mathrm{L}=2.75 \mathrm{~m}, \mathrm{H}=2.35 \mathrm{~m}$ ) after inserting three metallic hemispheres (Radius $0.4 \mathrm{~m})$.

induced by measurement noise. The use of a new resonance extraction method is proposed in this paper along with specific treatment to discriminate physical modes from spurious ones. In this approach used in the time domain, the transmission coefficient is modeled as an exponential sum corresponding to the cavity modes. However, this might lead to partially erroneous results as some spurious poles can be extracted by the numerical method. Indeed, such extraction methods are usually very sensitive to the noise and also to some internal parameters such as the number of poles to be extracted and the considered time window. Thus, we suggest studying the stability of each mode versus time in order to ensure that the poles are genuine. The Matrix Pencil Method (MPM) [7] is used throughout the paper as the numerical method to analyze the modes resonating inside the MSRC. Indeed, the MPM is already widely used in radar and antenna domains (in order to identify an arbitrary object by its complex resonances (poles) [8]) and presents good accuracy and robustness to the noise. The aim of this paper is thus twofold: on the one hand, to analyze the accuracy of the MPM to extract the modes resonating inside a MSRC, and on the other hand, to introduce some new tools based on the increasing time-window technique [9] to discriminate the true modes from the spurious ones.

Section II briefly introduces the MPM while the section III presents its application on a transmission coefficient measured inside a MSRC and focuses on the true pole discrimination process. 


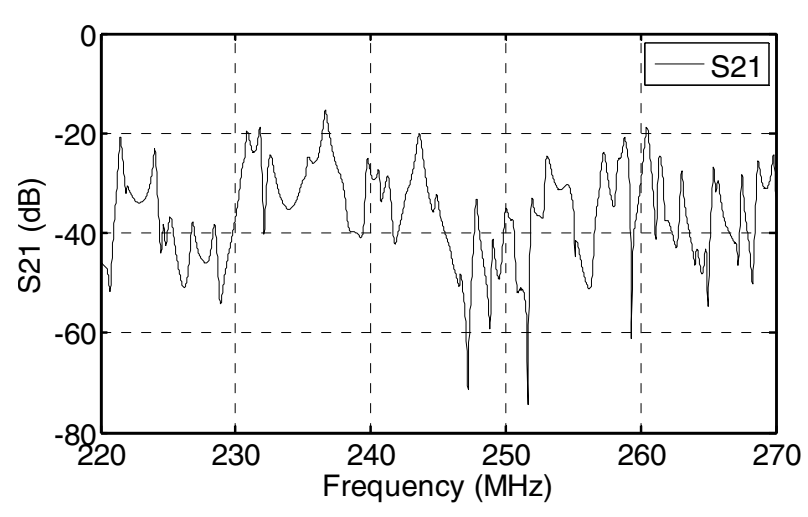

Fig. 2. S21 parameter measured inside the reverberation chamber as a function of the frequency.

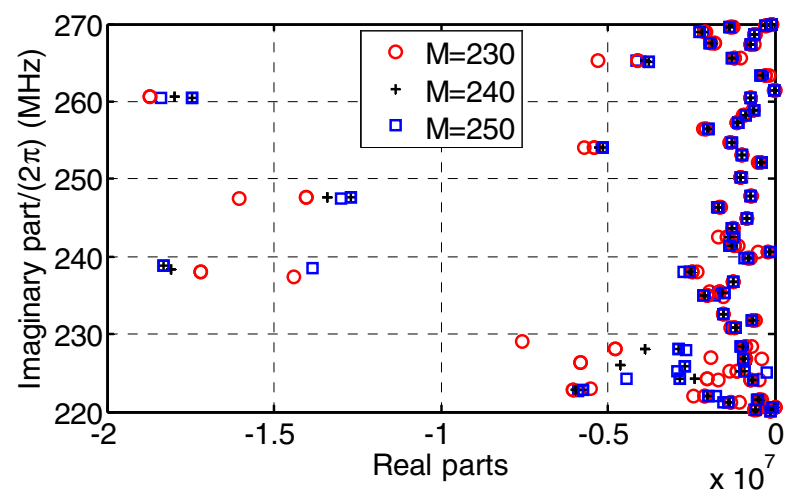

Fig. 3. Poles (modes) extracted using the MPM with several values of $\mathrm{M}$ in the complex plane. Only poles having positive imaginary parts and related to resonances in the $220-270 \mathrm{MHz}$ range are drawn.

\section{The MATRIX PENCIL Method}

The Matrix Pencil Method (MPM) allows any time signal $y(t)$ to be modeled as an exponential sum as:

$$
y(t)=\sum_{m=1}^{M} R_{m} e^{s_{m} t}
$$

where $s_{m}$ is the $\mathrm{m}^{\text {th }}$ complex pole, $R_{m}$ its corresponding residue (complex magnitude) and $M$ the number of poles to be extracted. The conjugate complex of each pole $s_{m}$ is also a pole, and poles can be written as $s_{m}=\alpha_{m} \pm j 2 \pi f_{m}$, with $\alpha_{m}$ the negative damping coefficient and $f_{m}$ the resonance frequency. The MPM is a two-steps process. First, a Singular Value Decomposition is performed on the Hankel matrix constructed using the input data vector. The pole extraction is then reduced to a generalized eigenvalue problem [7].

\section{MOdEs INSIDE THE REVERBERATION CHAMBER}

This section presents the application of the MPM on measurements performed inside the MSRC of ESYCOM laboratory presented in Fig. 1. It consists in an industrial reverberation chamber equipped with a vertical mode stirrer and geometrically modified by fixing three metallic hemispheres on adjacent cavity walls. In previous studies, we showed that this simple geometrical modification, which has

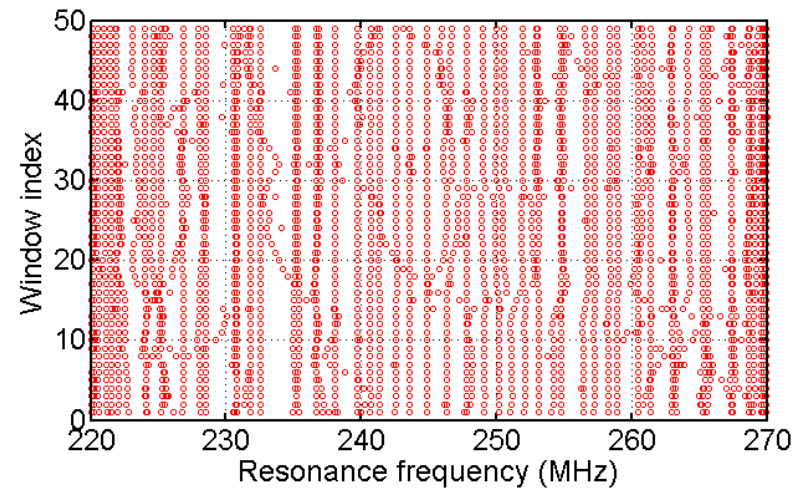

Fig. 4. Resonance frequencies (imaginary parts of the poles divided by $2 \pi$ ) extracted using the MPM applied on several time windows.

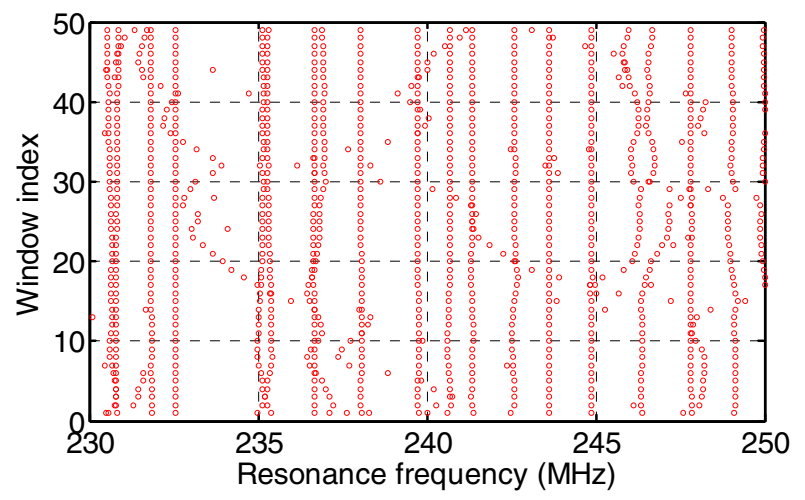

Fig. 5. Zoomed view of Fig. 4.

been inspired by the study of chaotic cavities, improves the MSRC performances [3]. The transmission coefficient between two small antennas located inside the MSRC has been measured using a Vector Network Analyzer in the 220$270 \mathrm{MHz}$ frequency range and over a whole stirrer rotation for a $1^{\circ}$ rotation step. According to the generalized Weyl formula [10], 52 resonance modes are expected on this frequency range.

The transmission coefficient measured at the first stirrer position (Fig. 2) shows the modal overlap (defined as the ratio of the mean resonance width and the mean distance between resonances and of 0.4 in this case) makes difficult the determining of the 52 resonance frequencies.

In order to obtain a time-domain signal, an Inverse Fast Fourier Transform (IFFT) is performed on the measured frequency-domain S21 parameter. In order to keep data with the highest signal-to-noise ratio, the time-domain S21 is truncated after $2 \mu \mathrm{s}$ as it includes $98 \%$ of the total power. The MPM is then applied using different values of $M$ (number of poles to be extracted) from 230 to 250. Extracted poles are presented in Fig. 3 in the complex plane. As the conjugate complex of each pole is also extracted, only the positive imaginary parts are presented here. Although 230 to 250 poles are extracted by the MPM (i.e. 115 to 125 modes), only 62 modes resonate in the considered frequency range (220-270 MHz). 
We observe that the poles with high real parts are not extracted exactly the same way according to $M$. These rapidly vanishing modes are indeed difficult to precisely characterize. However, the other ones (real parts closer to 0) appear to be the same whatever $M$. The poles present overall a good stability as a function of the parameter $M$ of the MPM. It has to be noticed that $M$ needs to be overestimated in order to ensure the extraction of all the cavity modes.

As only 52 modes actually resonate in this cavity in the measure frequency range, one needs to discriminate the true poles from the spurious ones, i.e. due to the measurement noise. The proposed approach consists of windowing the time S21, applying the MPM on this specific window, increasing the considered window of a few samples and repeating the process. Thus, each window begins at the same time but ends at a slightly different one. In this way, the poles related to a cavity mode should be extracted the same way whatever the window while the poles related to the measurement noise should vary since the actual data is not exactly the same in each window.

The imaginary parts of each pole are presented in Fig. 4 in terms of resonance frequencies as a function of the window index in the $220-270 \mathrm{MHz}$ frequency range. We observe that some resonant frequencies are very stable according to the window index while some others differ significantly. A zoom on the $230-250 \mathrm{MHz}$ frequency range is presented in Fig. 5. As an example, the poles whose resonant frequencies are $232 \mathrm{MHz}$ and $232.5 \mathrm{MHz}$ are very stable while the poles located in the $233-234 \mathrm{MHz}$ area are totally different from one window to another. Although some poles are not perfectly stable according to the window, they present an overall stability that allows considering those ones as true poles. Among the approximately 62 modes obtained from each window in the considered frequency range, 50 modes are stable while modifying the window length and can be thus considered as true poles. The other 12 modes vary from one window to another and present thus no physical meaning and can be considered as spurious modes.

\section{CONCLUSION}

The Matrix Pencil Method has been applied on frequency-domain S21 measurements performed inside a mode-stirred reverberation chamber and this method is able to extract the resonances that occur inside the cavity. A technique based on an increasing time-window has been introduced and allows extracting 50 out of the 52 modes that actually resonate inside the cavity with good accuracy. The presented technique is thus very relevant in order to discriminate the true poles from the spurious ones in a cavity mode extraction context.

\section{REFERENCES}

[1] P. S. Kildal, K. Rosengren, J. Byun and J. Lee, "Definition of effective diversity gain and how to measure it in a reverberation chamber," Microw. Opt. Technol. Lett., vol. 34, no. 1, pp. 56-59, July 2002.

[2] IEC 61000-4-21: Electromagnetic Compatibility (EMC)-Part 4-21: Testingand Measurement Techniques-Reverberation chamber test methods, International Electrotechnical Commission, IEC SC77BCISPR/A JWG REV, Geneva, Switzerland, Aug. 2003.

[3] J.-B. Gros, U. Kuhl, O. Legrand, F. Mortessagne, O. Picon, and E. Richalot, "Statistics of the electromagnetic response of a chaotic reverberation chamber", Advanced Electromagnetics, vol. 4, no. 2, pp. 38-43, 2015.

[4] E. Richalot; K. Selemani, J.B. Gros, O. Picon, O. Legrand, S. GrivetTalocia, and F. Mortessagne, "Criterion based on resonant frequencies distributions for reverberation chamber characterization", Int. Conf. on Electromag. in Advanced Applications (ICEAA), Sept. 2015.

[5] U. Kuhl, R. Höhmann, J. Main, and H.-J. Stöckmann, "Resonance widths in open microwave cavities studied by harmonic inversion", Phyical Review Letters, vol. 100, no 25, pp. 254101, Jun. 2008.

[6] S. Grivet-Talocia and M. Bandinu, "Improving the convergence of Vector Fitting for equivalent circuit extraction from noisy frequency responses," IEEE Trans. Electromag. Compat, vol. 48, pp. 104-120, Feb. 2006.

[7] T.K. Sarkar and O. Pereira, "Using the Matrix Pencil method to estimate the parameters of a sum of complex exponentials," IEEE Antennas and Propagation Magazine, vol. 37, pp 45-55, Feb. 1995.

[8] F. Sarrazin, J. Chauveau, P. Pouliguen, P. Potier and A. Sharaiha, "Accuracy of the singularity expansion method in time and frequency domains to characterize antennas in presence of noise," IEEE Transactions on Antennas and Propagation, vol. 62, no 3, pp 12611269, Mar. 2014

[9] F. Sarrazin, P. Pouliguen, A. Sharaiha, J. Chauveau and P. Potier, "Window increasing technique to discriminate mathematical and physical resonant poles extracted from antenna response," IET Electronics Letter, vol. 50, no. 5, pp. 343-344, 2014.

[10] R. Balian and C. Bloch, "Distribution of eigenfrequencies for the wave equation in a finite domain. II. Electromagnetic field. Riemannian spaces,” Ann. Phys., vol. 64, pp. 271-307, 1971. 